\title{
Global Behaviors and Optimal Harvesting of a Class of Impulsive Periodic Logistic Single-Species System with Continuous Periodic Control Strategy
}

\author{
JinRong Wang, ${ }^{1}$ X. Xiang, ${ }^{2}$ and W. Wei ${ }^{2}$ \\ ${ }^{1}$ College of Computer Science and Technology, Guizhou University, Guiyang, Guizhou 550025, China \\ ${ }^{2}$ College of Science, Guizhou University, Guiyang, Guizhou 550025, China \\ Correspondence should be addressed to JinRong Wang, wjr9668@126.com
}

Received 30 September 2008; Accepted 17 December 2008

Recommended by Wenming Zou

Global behaviors and optimal harvesting of a class of impulsive periodic logistic single-species system with continuous periodic control strategy is investigated. Four new sufficient conditions that guarantee the exponential stability of the impulsive evolution operator introduced by us are given. By virtue of exponential stability of the impulsive evolution operator, we present the existence, uniqueness and global asymptotical stability of periodic solutions. Further, the existence result of periodic optimal controls for a Bolza problem is given. At last, an academic example is given for demonstration.

Copyright (c) 2008 JinRong Wang et al. This is an open access article distributed under the Creative Commons Attribution License, which permits unrestricted use, distribution, and reproduction in any medium, provided the original work is properly cited.

\section{Introduction}

In population dynamics, the optimal management of renewable resources has been one of the interesting research topics. The optimal exploitation of renewable resources, which has direct effect on their sustainable development, has been paid much attention [1-3]. However, it is always hoped that we can achieve sustainability at a high level of productivity and good economic profit, and this requires scientific and effective management of the resources.

Single-species resource management model, which is described by the impulsive periodic logistic equations on finite-dimensional spaces, has been investigated extensively, no matter how the harvesting occurs, continuously [1,4] or impulsively [5-7]. However, the associated single-species resource management model on infinite-dimensional spaces has not been investigated extensively.

Since the end of last century, many authors including Professors Nieto and Hernández pay great attention on impulsive differential systems. We refer the readers to [8-22]. 
Particulary, Doctor Ahmed investigated optimal control problems [23, 24] for impulsive systems on infinite-dimensional spaces. We also gave a series of results [25-34] for the firstorder (second-order) semilinear impulsive systems, integral-differential impulsive system, strongly nonlinear impulsive systems and their optimal control problems. Recently, we have investigated linear impulsive periodic system on infinite-dimensional spaces. Some results [35-37] including the existence of periodic $P C$-mild solutions and alternative theorem, criteria of Massera type, asymptotical stability and robustness against perturbation for a linear impulsive periodic system are established.

Herein, we devote to studying global behaviors and optimal harvesting of the generalized logistic single-species system with continuous periodic control strategy and periodic impulsive perturbations:

$$
\begin{gathered}
\frac{\partial}{\partial t} x(t, y)=A(y, D) x(t, y)+f(t, y)+C(t) u(t, y), \quad u \in U_{a d}, y \in \Omega, t>0, t \neq \tau_{k}, k \in \mathbb{Z}_{0}^{+}, \\
x(t, y)=0, \quad y \in \partial \Omega, t>0, \\
\Delta x(t, y)=\Delta x(t+0, y)-\Delta x(t, y)=B_{k} x(t, y)+c_{k}, \quad y \in \Omega, t=\tau_{k}, k \in \mathbb{Z}_{0}^{+} .
\end{gathered}
$$

On infinite-dimensional spaces, where $x(t, y)$ denotes the population number of isolated species at time $t$ and location $y, \Omega \subset \mathbb{R}^{2}$ is a bounded domain and $\partial \Omega \in C^{2}$, operator $A(y, D)=\sum_{|\alpha| \leq 4} a_{\alpha}(y) D^{\alpha}$. The coefficients $a_{\alpha}(y),(y \in \bar{\Omega}, t \geq 0)$ are sufficiently smooth functions of $y$ in $\bar{\Omega}$, where $\alpha=\left(\alpha_{1}, \alpha_{2}\right), \alpha_{i}>0, i=1,2,|\alpha|=\sum_{i=1}^{2} \alpha_{i}$ and $y^{\alpha}=y_{1}^{\alpha} y_{2}^{\alpha}$, $y=\left(y_{1}, y_{2}\right) \in \Omega$. Denoting $D_{i}=\left(\partial / \partial y_{i}\right)(i=1,2), D=\left(D_{1}, D_{2}\right)$, then $D^{\alpha}=D_{1}^{\alpha_{1}} D_{2}^{\alpha_{2}} \cdot f(t, y)$ is related to the periodic change of the resources maintaining the evolution of the population and the periodic control policy $u \in U_{a d}$, where $U_{a d}$ is a suitable admissible control set. Time sequence $0=\tau_{0}<\tau_{1}<\cdots<\tau_{k} \cdots$ and $\tau_{k} \rightarrow \infty$ as $k \rightarrow \infty, \Delta x\left(\tau_{k}, y\right)$ denote mutation of the isolate species at time $\tau_{k}$ where $k \in \mathbb{Z}_{0}^{+}$.

Suppose $X$ is a Banach space and $Y$ is a separable reflexive Banach space. The objective functional is given by

$$
\tilde{J}(u)=\int_{0}^{T_{0}} \int_{\Omega} l(t, x(t, y, u), u(t, y)) d y d t+\int_{\Omega} \Psi\left(x\left(T_{0}, y, u\right)\right) d y,
$$

where $l:\left[0, T_{0}\right] \times X \times Y \rightarrow \mathbb{R} \cup\{\infty\}$ is Borel measurable, $\Psi: X \rightarrow \mathbb{R}$ is continuous, and nonnegative and $x(\cdot, y, u)$ denotes the $T_{0}$-periodic $P C$-mild solution of system (1.1) at location $y$ and corresponding to the control $u \in U_{a d}$. The Bolza problem $(\tilde{P})$ is to find $u^{0} \in U_{a d}$ such that $\widetilde{J}\left(u^{0}\right) \leq \widetilde{J}(u)$ for all $u \in U_{a d}$.

Suppose that $f\left(t+T_{0}, y\right)=f(t, y), C\left(t+T_{0}\right)=C(t), u\left(t+T_{0}, y\right)=u(t, y), t \geq 0$ and $T_{0}$ is the least positive constant such that there are $\delta \tau_{k} \mathrm{~s}$ in the interval $\left(0, T_{0}\right)$, and $\tau_{k+\delta}=\min \{\tau \in$ $\left.\widetilde{D} \mid \tau \geq \tau_{k}+T_{0}\right\}$, where $\widetilde{D}=\left\{\tau_{k} \mid \tau_{k+1}>\tau_{k}\right.$; for all $\left.k \in \mathbb{Z}_{0}^{+}\right\}, B_{k+\delta}=B_{k}, c_{k+\delta}=c_{k}, k \in \mathbb{Z}_{0}^{+}$. The first equation of system (1.1) describes the variation of the population number $x$ of the species in periodically continuous controlled changing environment. The second equation of system (1.1) shows that the species are isolated. The third equation of system (1.1) reflects the possibility of impulsive effects on the population.

Let $A(y, D)$ satisfy some properties (such as strongly elliptic) in $\Omega$ and set $D(A)$ (such as $\left.H^{2}(\Omega) \cap H_{0}^{1}(\Omega)\right)$. For every $x \in D(A)$ define $A x=A(y, D) x, A$ is the infinitesimal generator 
of a $C_{0}$-semigroup $\{T(t), t \geq 0\}$ on the Banach space $X$ (such as $H^{2}(\Omega)$ ). Define $\mathrm{x}(\cdot)(y)=$ $x(\cdot, y),(f)(\cdot)(y)=f(\cdot, y)$, and $u(\cdot)(y)=u(\cdot, y)$ then system (1.1) can be abstracted into the following controlled system:

$$
\begin{gathered}
\dot{x}(t)=A x(t)+f(t)+C(t) u(t), \quad u \in U_{a d}, t \neq \tau_{k}, \\
\Delta x\left(\tau_{k}\right)=B_{k} x\left(\tau_{k}\right)+c_{k}, \quad t=\tau_{k} .
\end{gathered}
$$

On the Banach space $X$, and the associated objective functional

$$
J(u)=\int_{0}^{T_{0}} l(t, x(t, u), u(t)) d t+\Psi\left(x\left(T_{0}, u\right)\right)
$$

where $x(\cdot, u)$ denotes the $T_{0}$-periodic $P C$-mild solution of system (1.3) corresponding to the control $u \in U_{a d}$. The Bolza problem (P) is to find $u^{0} \in U_{a d}$ such that $J\left(u^{0}\right) \leq J(u)$ for all $u \in U_{a d}$. The investigation of the system (1.3) cannot only be used to discuss the system (1.1), but also provide a foundation for research of the optimal control problems for semilinear impulsive periodic systems. The aim of this paper is to give some new sufficient conditions which will guarantee the existence, uniqueness, and global asymptotical stability of periodic $P C$-mild solutions for system (1.3) and study the optimal control problems arising in the system (1.3).

The paper is organized as follows. In Section 2, the properties of the impulsive evolution operator $\{S(\cdot, \cdot)\}$ are collected. Four new sufficient conditions that guarantee the exponential stability of the $\{S(\cdot, \cdot)\}$ are given. In Section 3 , the existence, uniqueness, and global asymptotical stability of $T_{0}$-periodic $P C$-mild solution for system (1.3) is obtained. In Section 4, the existence result of periodic optimal controls for the Bolza problem $(\mathrm{P})$ is presented. At last, an academic example is given to demonstrate our result.

\section{Impulsive periodic evolution operator and it's stability}

Let $X$ be a Banach space, $£(X)$ denotes the space of linear operators on $X ; £_{b}(X)$ denotes the space of bounded linear operators on $X . £_{b}(X)$ is the Banach space with the usual supremum norm. Denote $\tilde{D}=\left\{\tau_{1}, \ldots, \tau_{\delta}\right\} \subset\left[0, T_{0}\right]$ and define $P C\left(\left[0, T_{0}\right] ; X\right) \equiv\left\{x:\left[0, T_{0}\right] \rightarrow X \mid x\right.$ is continuous at $t \in\left[0, T_{0}\right] \backslash \tilde{D}, x$ is continuous from left and has right-hand limits at $\left.t \in \tilde{D}\right\}$ and $P C^{1}\left(\left[0, T_{0}\right] ; X\right) \equiv\left\{x \in P C\left(\left[0, T_{0}\right] ; X\right) \mid \dot{x} \in P C\left(\left[0, T_{0}\right] ; X\right)\right\}$.

Set

$$
\|x\|_{P C}=\max \left\{\sup _{t \in\left[0, T_{0}\right]}\|x(t+0)\|, \sup _{t \in\left[0, T_{0}\right]}\|x(t-0)\|\right\}, \quad\|x\|_{P C^{1}}=\|x\|_{P C}+\|\dot{x}\|_{P C} .
$$

It can be seen that endowed with the norm $\|\cdot\|_{P C}\left(\|\cdot\|_{P C^{1}}\right), P C\left(\left[0, T_{0}\right] ; X\right)\left(P C^{1}\left(\left[0, T_{0}\right] ; X\right)\right)$ is a Banach space. 
In order to investigate periodic solutions, we introduce the following two spaces:

$$
\begin{gathered}
L_{T_{0}}^{p}([0,+\infty) ; X) \equiv\left\{f:[0,+\infty) \longrightarrow X \mid f(t)=f\left(t+T_{0}\right),\right. \\
\left.\left(\int_{0}^{T_{0}}\|f(t)\|^{p} d t\right)^{1 / p}<+\infty \text { where } 1<p<+\infty\right\}, \\
P C_{T_{0}}([0,+\infty) ; X) \equiv\left\{x \in P C([0,+\infty) ; X) \mid x(t)=x\left(t+T_{0}\right), t \geq 0\right\} .
\end{gathered}
$$

Set

$$
\|f\|_{L_{T_{0}}^{p}}=\left(\int_{0}^{T_{0}}\|f(t)\|^{p} d t\right)^{1 / p}, \quad\|x\|_{P C_{T_{0}}}=\max \left\{\sup _{t \in\left[0, T_{0}\right]}\|x(t+0)\|, \sup _{t \in\left[0, T_{0}\right]}\|x(t-0)\|\right\} .
$$

It can be seen that endowed with the norm $\|\cdot\|_{L_{T_{0}}^{p}}\left(\|\cdot\|_{P C_{T_{0}}}\right), L_{T_{0}}^{p}([0,+\infty) ; X)\left(P C_{T_{0}}([0,+\infty) ; X)\right)$ is a Banach space.

We introduce assumption [H1].

[H1.1]: $A$ is the infinitesimal generator of a $C_{0}$-semigroup $\{T(t), t \geq 0\}$ on $X$ with domain $D(A)$.

[H1.2]: There exists $\delta$ such that $\tau_{k+\delta}=\min \left\{\tau \in \tilde{D} \mid \tau \geq \tau_{k}+T_{0}\right\}$, where $\tilde{D}=\left\{\tau_{k} \mid \tau_{k+1}>\right.$ $\left.\tau_{k} ; \forall k \in \mathbb{Z}_{0}^{+}\right\}$.

[H1.3]: For each $k \in \mathbb{Z}_{0}^{+}, B_{k} \in £_{b}(X), B_{k+\delta}=B_{k}$.

Under the assumption [H1], consider

$$
\begin{aligned}
\dot{x}(t) & =A x(t), \quad t \neq \tau_{k}, \\
\Delta x(t) & =B_{k} x(t), \quad t=\tau_{k},
\end{aligned}
$$

and the associated Cauchy problem

$$
\begin{gathered}
\dot{x}(t)=A x(t), \quad t \in\left[0, T_{0}\right] \backslash \tilde{D}, \\
\Delta x\left(\tau_{k}\right)=B_{k} x\left(\tau_{k}\right), \quad k=1,2, \ldots, \delta, \\
x(0)=\bar{x} .
\end{gathered}
$$

For every $\bar{x} \in X, D(A)$ is an invariant subspace of $B_{k}$, using ([38, Theorem 5.2.2, page 144$])$, step by step, one can verify that the Cauchy problem (2.5) has a unique classical 
solution $x \in P C^{1}\left(\left[0, T_{0}\right] ; X\right)$ represented by $x(t)=S(t, 0) \bar{x}$, where $S(\cdot, \cdot): \Delta=\{(t, \theta) \mid 0 \leq \theta \leq$ $\left.t \leq T_{0}\right\} \rightarrow £(X)$ given by

$$
S(t, \theta)=\left\{\begin{array}{lc}
T(t-\theta), & \tau_{k-1} \leq \theta \leq t \leq \tau_{k}, \\
T\left(t-\tau_{k}^{+}\right)\left(I+B_{k}\right) T\left(\tau_{k}-\theta\right), & \tau_{k-1} \leq \theta<\tau_{k}<t \leq \tau_{k+1}, \\
T\left(t-\tau_{k}^{+}\right)\left[\prod_{\theta<\tau_{j}<t}\left(I+B_{j}\right) T\left(\tau_{j}-\tau_{j-1}^{+}\right)\right]\left(I+B_{i}\right) T\left(\tau_{i}-\theta\right), \\
\tau_{i-1} \leq \theta<\tau_{i} \leq \cdots<\tau_{k}<t \leq \tau_{k+1} .
\end{array}\right.
$$

The operator $\{S(t, \theta),(t, \theta) \in \Delta\}$ is called impulsive evolution operator associated with $\{T(t), t \geq 0\}$ and $\left\{B_{k} ; \tau_{k}\right\}_{k=1}^{\infty}$.

The following lemma on the properties of the impulsive evolution operator $\{S(t, \theta),(t, \theta) \in \Delta\}$ associated with $\{T(t), t \geq 0\}$ and $\left\{B_{k} ; \tau_{k}\right\}_{k=1}^{\infty}$ is widely used in this paper.

Lemma 2.1. Let assumption [H1] hold. The impulsive evolution operator $\{S(t, \theta),(t, \theta) \in \Delta\}$ has the following properties.

(1) For $0 \leq \theta \leq t \leq T_{0}, S(t, \theta) \in £_{b}(X)$, there exists a $M_{T_{0}}>0$ such that $\sup _{0 \leq \theta \leq t \leq T_{0}}\|S(t, \theta)\| \leq M_{T_{0}}$.

(2) For $0 \leq \theta<r<t \leq T_{0}, r \neq \tau_{k}, S(t, \theta)=S(t, r) S(r, \theta)$.

(3) For $0 \leq \theta \leq t \leq T_{0}, n \in Z^{+}, S\left(t+n T_{0}, \theta+n T_{0}\right)=S(t, \theta)$.

(4) For $0 \leq \theta \leq t \leq T_{0}, n \in Z^{+}, S\left(t+n T_{0}, 0\right)=S(t, 0)\left[S\left(T_{0}, 0\right)\right]^{n}$.

(5) For $0 \leq \theta<t$, there exits $M \geq 1, \omega \in \mathbb{R}$ such that

$$
\|S(t, \theta)\| \leq M \exp \left\{\omega(t-\theta)+\sum_{\theta \leq \tau_{n}<t} \ln \left(M\left\|I+B_{n}\right\|\right)\right\} .
$$

Proof. (1) By assumption [H1.1], there exists a constant $\mathcal{C}_{T_{0}}>0$ such that $\sup _{t \in\left[0, T_{0}\right]}\|T(t)\|=$ $\mathcal{C}_{T_{0}}<\infty$. Using assumption [H1.3], it is obvious that $S(t, \theta) \in £_{b}(X)$, for $0 \leq \theta \leq t \leq T_{0}$. (2) By the definition of $C_{0}$-semigroup and the construction of $S(\cdot, \cdot)$, one can verify the result immediately. (3) By assumptions [H1.2], [H1.3], and elementary computation, it is easy to obtain the result. (4) For $0 \leq \theta \leq t \leq T_{0}, n \in Z^{+}$, by virtue of (3) again and again, we arrive at

$$
\begin{aligned}
S\left(t+n T_{0}, 0\right) & =S\left(t+n T_{0}, T_{0}\right) S\left(T_{0}, 0\right)=S\left(t+(n-1) T_{0}, 0\right) S\left(T_{0}, 0\right) \\
& =S\left(t+(n-1) T_{0}, T_{0}\right) S\left(T_{0}, 0\right) S\left(T_{0}, 0\right)=\left(\left(t+(n-2) T_{0}, 0\right)\left[S\left(T_{0}, 0\right)\right]^{2}\right. \\
& \ldots \\
& =S(t, 0)\left[S\left(T_{0}, 0\right)\right]^{n} .
\end{aligned}
$$


(5) Without loss of generality, for $\tau_{i-1} \leq \theta<\tau_{i} \leq \cdots<\tau_{k}<t \leq \tau_{k+1}$,

$$
\begin{aligned}
\|S(t, \theta)\| & =\left\|T\left(t-\tau_{k}^{+}\right)\right\|\left\|I+B_{k}\right\|\left\|T\left(\tau_{k}-\tau_{k-1}^{+}\right)\right\| \cdots\left\|I+B_{i}\right\|\left\|T\left(\tau_{i}-\theta\right)\right\| \\
& \leq M e^{\omega\left(t-\tau_{k}^{+}\right)}\left[\prod_{n=i+1}^{k}\left\|I+B_{n}\right\| M e^{\left(\tau_{n}-\tau_{n-1}^{+}\right)}\right]\left\|I+B_{i}\right\| M e^{\omega\left(\tau_{i}-\theta\right)} \\
& \leq M \exp \left\{\omega(t-\theta)+\sum_{\theta \leq \tau_{n}<t} \ln \left(M\left\|I+B_{n}\right\|\right)\right\} .
\end{aligned}
$$

This completes the proof.

In order to study the asymptotical properties of periodic solutions, it is necessary to discuss the exponential stability of the impulsive evolution operator $\{S(t, \theta), t \geq \theta \geq 0\}$. We first give the definition of exponential stable for $\{S(t, \theta), t \geq \theta \geq 0\}$.

Definition 2.2. $\{S(t, \theta), t \geq \theta \geq 0\}$ is called exponentially stable if there exist $K \geq 0$ and $v>0$ such that

$$
\|S(t, \theta)\| \leq K e^{-v(t-\theta)}, \quad t>\theta \geq 0 .
$$

Assumption [H2]: $\{T(t), t \geq 0\}$ is exponentially stable, that is, there exist $K_{0}>0$ and $v_{0}>0$ such that

$$
\|T(t)\| \leq K_{0} e^{-v_{0} t}, \quad t>0 .
$$

An important criteria for exponential stability of a $C_{0}$-semigroup is collected here.

Lemma 2.3 (see [38, Lemma 7.2.1]). Let $\{T(t), t \geq 0\}$ be a $C_{0}$-semigroup on $X$, and let $A$ be its infinitesimal generator. Then the following assertions are equivalent:

(1) $\{T(t), t \geq 0\}$ is exponentially stable.

(2) For every $x \in X$ there exits a positive constants $\gamma_{x}<\infty$ such that

$$
\int_{0}^{\infty}\|T(t) x\|^{p} d t<\gamma_{x}<\infty, \quad x \in X, t>0, \text { for some } p, 1 \leq p<\infty
$$

Next, four sufficient conditions that guarantee the exponential stability of impulsive evolution operator $\{S(t, \theta), t \geq \theta \geq 0\}$ are given.

Lemma 2.4. Assumptions [H1] and [H2] hold. There exists $0<\lambda<v_{0}$ such that

$$
\prod_{k=1}^{\delta}\left(K_{0}\left\|I+B_{k}\right\|\right) e^{-\lambda T_{0}}<1
$$

Then, $\{S(t, \theta), t \geq \theta \geq 0\}$ is exponentially stable. 
Proof. Without loss of generality, for $\tau_{i-1} \leq \theta<\tau_{i} \leq \cdots<\tau_{k}<t \leq \tau_{k+1}$, we have

$$
\|S(t, \theta)\| \leq K_{0} e^{-\left(v_{0}-\lambda\right)(t-\theta)}\left[\prod_{\theta<\tau_{k}<t}\left(K_{0}\left\|I+B_{k}\right\|\right) e^{-\lambda(t-\theta)}\right] .
$$

Suppose $t \in\left(n T_{0},(n+1) T_{0}\right]$ and let $b=\max _{s \in\left[0, T_{0}\right]} \prod_{0<\tau_{k}<s}\left\{K_{0}\left\|I+B_{k}\right\|\right\}$. Then,

$$
\begin{aligned}
\prod_{\theta<\tau_{k}<t}\left(K_{0}\left\|I+B_{k}\right\|\right) e^{-\lambda(t-\theta)} & \leq \prod_{0 \leq \tau_{k}<n T_{0}}\left(K_{0}\left\|I+B_{k}\right\|\right) e^{-\lambda n T_{0}} b e^{\lambda \theta} \\
& \leq \prod_{k=1}^{\delta}\left(K_{0}\left\|I+B_{k}\right\|\right)^{n} e^{-\lambda n T_{0}} b e^{\lambda \theta} \\
& =\left[\prod_{k=1}^{\delta}\left(K_{0}\left\|I+B_{k}\right\|\right) e^{-\lambda T_{0}}\right]^{n} b e^{\lambda \theta} \\
& <b e^{\lambda \theta} .
\end{aligned}
$$

Let $K=K_{0} b e^{\lambda \theta}>0$ and $v=v_{0}-\lambda>0$, then we obtain $\|S(t, \theta)\| \leq K e^{-v(t-\theta)}, t>\theta \geq 0$.

Lemma 2.5. Assume that assumption [H1] holds. Suppose

$$
0<\mu_{1}=\inf _{k=1,2, \ldots, \delta}\left(\tau_{k}-\tau_{k-1}\right) \leq \sup _{k=1,2, \ldots, \delta}\left(\tau_{k}-\tau_{k-1}\right)=\mu_{2}<\infty
$$

If there exists $\alpha>0$ such that

$$
\omega+\frac{1}{\mu} \ln \left(M\left\|I+B_{k}\right\|\right) \leq-\alpha<0,
$$

for $k=1,2, \ldots, \delta$, where

$$
\mu= \begin{cases}\mu_{1}, & \alpha+\omega<0 \\ \mu_{2}, & \alpha+\omega \geq 0\end{cases}
$$

Then, $\{S(t, \theta), t \geq \theta \geq 0\}$ is exponentially stable.

Proof. It comes from (2.17) that

$$
\ln \left(M\left\|I+B_{k}\right\|\right) \leq-\mu(\alpha+\omega)<0, \quad k=1,2, \ldots, \delta .
$$


Further,

$$
\sum_{\theta \leq \tau_{k}<t} \ln \left(M\left\|I+B_{k}\right\|\right) \leq-\sum_{\theta \leq \tau_{k}<t} \mu(\alpha+\omega)=-\mu(\alpha+\omega) N(\theta, t)
$$

where $N(\theta, t)$ is denoted the number of impulsive points in $[\theta, t)$.

For $\tau_{i-1} \leq \theta<\tau_{i} \leq \cdots<\tau_{k}<t \leq \tau_{k+1}$, by (2.16), we obtain the following two inequalities:

$$
t-\theta \geq(N(\theta, t)-1) \mu_{1}, \quad t-\theta \leq(N(\theta, t)+1) \mu_{2} .
$$

This implies

$$
\mu_{1}(N(\theta, t)-1) \leq t-\theta \leq \mu_{2}(N(\theta, t)+1)
$$

that is,

$$
\frac{1}{\mu_{2}}(t-\theta)-1 \leq N(\theta, t) \leq \frac{1}{\mu_{1}}(t-\theta)+1 .
$$

Then,

$$
-\mu(\alpha+\omega) N(\theta, t) \leq-(\alpha+\omega)(t-\theta)+\mu|\alpha+\omega|
$$

Thus, we obtain

$$
\omega(t-\theta)+\sum_{\theta \leq \tau_{k}<t} \ln \left(M\left\|I+B_{k}\right\|\right) \leq-\alpha(t-\theta)+\mu|\alpha+\omega| .
$$

By (5) of Lemma 2.1, let $K=M e^{\mu|\alpha+\omega|}>0, v=\alpha>0,\|S(t, \theta)\| \leq K e^{-v(t-\theta)}, t>\theta \geq 0$.

Lemma 2.6. Assume that assumption [H1] holds. The limit

$$
\lim _{T_{0} \rightarrow \infty} \frac{N\left(\theta, \theta+T_{0}\right)}{T_{0}} \text { exists and is equal to } \frac{\delta}{T_{0}} \equiv p \text { is finite. }
$$

Suppose there exists $\gamma>0$ such that

$$
\omega+p \ln \left(M\left\|I+B_{k}\right\|\right) \leq-\gamma<0, \quad k=1,2, \ldots, \delta .
$$

Then, $\{S(t, \theta), t \geq \theta \geq 0\}$ is exponentially stable.

Proof. Let $t, \theta \in \mathbb{R}^{+}$with $t>\theta$. It comes from

$$
\lim _{T_{0} \rightarrow \infty} \frac{N\left(\theta, \theta+T_{0}\right)}{T_{0}}=\frac{\delta}{T_{0}} \equiv p
$$


that there exits a $h>0$ enough small such that

$$
\left|\frac{N(\theta, t)}{t-\theta}-p\right|<p h
$$

that is,

$$
(1-h)(t-\theta)<\frac{N(\theta, t)}{p}<(1+h)(t-\theta)
$$

From (2.27), we know that

$$
\sum_{\theta<\tau_{k}<t} \ln \left(M\left\|I+B_{k}\right\|\right) \leq-\sum_{\theta<\tau_{k}<t} \frac{1}{p}(\gamma+\omega)=-\frac{N(\theta, t)}{p}(\gamma+\omega) .
$$

Then, we have

$$
-\frac{N(\theta, t)}{p}(\gamma+\omega) \leq\left\{\begin{array}{ll}
-\frac{(1+h)}{p}(t-\theta)(\gamma+\omega), & \gamma+\omega<0 \\
-\frac{(1-h)}{p}(t-\theta)(\gamma+\omega), & \gamma+\omega \geq 0
\end{array}=-[(\gamma+\omega)-h|\gamma+\omega|](t-\theta) .\right.
$$

Hence,

$$
(\omega+h)(t-\theta)+\sum_{\theta<\tau_{k}<t} \ln \left(M\left\|I+B_{k}\right\|\right) \leq-[\gamma-h(1+|\gamma+\omega|)](t-\theta)
$$

Here, we only need to choose $h>0$ small enough such that $\gamma-h(1+|\gamma+\omega|)>0$, by (5) of Lemma 2.1 again, let $K=M>0, v=\gamma-h(1+|\gamma+\omega|)>0$, we have $\|S(t, \theta)\| \leq K e^{-v(t-\theta)}, t>$ $\theta \geq 0$.

Lemma 2.7. Assume that assumption [H1] holds. For some $p, 1 \leq p<+\infty$,

$$
\begin{gathered}
\int_{0}^{\infty}\|S(t, \theta) \xi\|^{p} d t<\infty, \quad \xi \in X, t>\theta \geq 0, \theta \text { is fixed } \\
\sum_{\theta \leq \tau_{k}<t}\left\|I+B_{k}\right\| \text { is convergent. }
\end{gathered}
$$

Imply the exponential stability of $\{S(t, \theta), t \geq \theta \geq 0\}$.

Proof. It comes from the continuity of $t \rightarrow T(t) \xi$, the inequality

$$
\|S(t, \theta)\| \leq\left(M^{2} \sum_{\theta \leq \tau_{k}<t}\left\|I+B_{k}\right\|\right) e^{\omega(t-\theta)}
$$


and the boundedness of $B_{k}, \sum_{\theta \leq \tau_{k}<t}\left\|I+B_{k}\right\|$ are convergent, that $\lim _{t \rightarrow \infty} S(t, \theta) \xi=0$ for every $\xi \in X$ and fixed $\theta \geq 0$. This shows that $S(t, \theta) \xi$ is bounded for each $\xi \in X$ and fixed $\theta \geq$ 0 and hence, by virtue of uniform boundedness principle, there exists a constant $M_{2} \geq 1$ such that $\|S(t, \theta)\| \leq M_{2}$ for all $t>\theta \geq 0$. Let $\perp$ denote the operator given by $(\llcorner x)(t)=$ $S(t, \theta) x, x \in X$ and $\theta$ is fixed. Clearly, $\mathcal{L}$ is defined every where on $X$ and by assumption it maps $X \rightarrow L^{p}((0, \infty) ; X)$ and it is a closed operator. Hence, by closed graph theorem, it is a bounded linear operator from $X$ to $L^{p}((0, \infty) ; X)$. Thus, there exits a constant $M_{3}>0$ such that $\|\mathcal{L} x\|_{L^{p}((0, \infty) ; X)} \leq M_{3}$ for all $x \in$ and $t>\theta \geq 0, \theta$ is fixed.

Let $0<\kappa<M_{2}^{-1}, \xi \in X$ and $t \geq 0$ and define $\tau \equiv \tau(\kappa, \xi)$ as

$$
\tau=\sup \{t \geq 0:\|S(s, \theta) \xi\| \geq \kappa\|\xi\| \forall 0 \leq \theta<s \leq t\}
$$

Then,

$$
\tau(\kappa\|\xi\|)^{p} \leq \int_{0}^{\tau}\|S(t, \theta) \xi\|^{p} d t \leq \int_{0}^{\infty}\|S(t, \theta) \xi\|^{p} d t=\|\mathcal{L} \xi\|_{L_{p}((0, \infty) ; X)}^{p} \leq\left(M_{3}\|\xi\|^{p},\right.
$$

and hence,

$$
\tau \leq\left(\frac{M_{3}}{\kappa}\right)^{p} \equiv t_{0} .
$$

Thus, for $t>\left(t_{0}+\theta\right) \neq \tau_{k}$,

$$
\|S(t, \theta) \xi\| \leq\|S(t, t-\tau) S(t-\tau, \theta) \xi\| \leq M_{2} \kappa\|\xi\| \equiv \beta\|\xi\|,
$$

where $\beta=M_{2} \mathcal{K}<1$. Fix $t_{1}=N_{0} T_{0}>t_{0}+\theta$. Then, for any $t \in[0, \infty)$ we can write $t-\theta=n t_{1}+s$ for some $n \in \mathbb{N}_{0}$ and $s \in\left[0, t_{1}\right)$ and we have

$$
\|S(t, \theta) \xi\|=\left\|S\left(n N_{0} T_{0}+s, \theta\right) \xi\right\|=\|S(s, \theta)\|\left\|S\left(T_{0}, 0\right) \xi\right\|^{n N_{0}} \leq M_{2} e^{n N_{0} \ln \beta}\|\xi\|=M_{1} e^{-v t}\|\xi\|,
$$

where $M_{1}=M_{2} \beta^{s / t_{1}}$ and $v=-\left(\ln \beta / t_{1}\right)$. Since $\beta<1$, this shows that our result.

\section{Periodic solutions and global asymptotical stability}

Consider the following controlled system:

$$
\begin{gathered}
\dot{x}(t)=A x(t)+f(t)+C(t) u(t), \quad u \in U_{a d}, t \neq \tau_{k}, \\
\Delta x\left(\tau_{k}\right)=B_{k} x\left(\tau_{k}\right)+c_{k}, \quad t=\tau_{k},
\end{gathered}
$$


and the associated Cauchy problem

$$
\begin{gathered}
\dot{x}(t)=A x(t)+f(t)+C(t) u(t), \quad u \in U_{a d}, t \in\left[0, T_{0}\right] \backslash \tilde{D}, \\
\Delta x\left(\tau_{k}\right)=B_{k} x\left(\tau_{k}\right)+c_{k}, \quad k=1,2, \ldots, \delta, \\
x(0)=\bar{x} .
\end{gathered}
$$

In addition to assumption [H1], we make the following assumptions:

[H3]: $f:\left[0, T_{0}\right] \rightarrow X$ is measurable and $f\left(t+T_{0}\right)=f(t)$ for $t \geq 0$.

[H4]: For each $k \in \mathbb{Z}^{+}$, there exists $\delta \in \mathbb{N}$ and $c_{k} \in X, c_{k+\delta}=c_{k}$.

[H5]: $U(\cdot):\left[0, T_{0}\right] \rightarrow 2^{Y} \backslash\{\varnothing\}$ has bounded, closed, and convex values and is graph measurable, $U(\cdot) \subseteq \Omega$ and $\Omega$ are bounded, where $Y$ is a separable reflexive Banach space.

[H6]: Operator $C \in £_{\infty}\left(\left[0, T_{0}\right] ; £(Y, X)\right)$ and $C\left(t+T_{0}\right)=C(t)$, for $t \geq 0$. Obviously, $C$ : $L^{p}\left(\left[0, T_{0}\right] ; Y\right) \rightarrow L^{p}\left(\left[0, T_{0}\right] ; X\right)(1<p<+\infty)$.

Denote the set of admissible controls

$$
U_{a d}=\left\{u(\cdot):\left[0, T_{0}\right] \longrightarrow Y \text { measurable } \mid u\left(t+T_{0}\right)=u(t), u(t) \in U(t) \text { a.e. for } t \geq 0\right\}
$$

Obviously, $U_{a d} \neq \varnothing$ and $U_{a d} \subseteq L^{p}\left(\left[0, T_{0}\right] ; Y\right)(1<p<\infty), U_{a d}$ is bounded, convex, and closed.

We introduce $P C$-mild solution of Cauchy problem (3.2) and $T_{0}$-periodic $P C$-mild solution of system (3.1).

Definition 3.1. A function $x \in P C\left(\left[0, T_{0}\right] ; X\right)$, for finite interval $\left[0, T_{0}\right]$, is said to be a $P C$-mild solution of the Cauchy problem (3.2) corresponding to the initial value $\bar{x} \in X$ and $u \in U_{a d}$ if $x$ is given by

$$
x(t, \bar{x}, u)=S(t, 0) \bar{x}+\int_{0}^{t} S(t, \theta)[f(\theta)+C(\theta) u(\theta)] d \theta+\sum_{0 \leq \tau_{k}<t} S\left(t, \tau_{k}^{+}\right) c_{k} .
$$

A function $x \in P C([0,+\infty) ; X)$ is said to be a $T_{0}$-periodic $P C$-mild solution of system (3.1) if it is a PC-mild solution of Cauchy problem (3.2) corresponding to some $\bar{x}$ and $x(t+$ $\left.T_{0}, \bar{x}, u\right)=x(t, \bar{x}, u)$ for $t \geq 0$.

Theorem 3.2. Assumptions [H1], [H3], [H4], [H5], and [H6] hold. Suppose $\{S(t, \theta), t \geq \theta \geq 0\}$ is exponentially stable, for every $u \in U_{a d}$, system (3.1) has a unique $T_{0}$-periodic PC-mild solution:

$$
x_{T_{0}}(t, \bar{x}, u)=S(t, 0) \bar{x}+\int_{0}^{t} S(t, \theta) g_{u}(\theta) d \theta+\sum_{0 \leq \tau_{k}<t} S\left(t, \tau_{k}^{+}\right) c_{k} \equiv P\left(g_{u}, c_{k}\right)(t),
$$


where $g_{u}(\cdot)=f(\cdot)+C(\cdot) u(\cdot) \in L_{T_{0}}^{p}([0, \infty) ; X)$,

$$
\bar{x}=\left[I-S\left(T_{0}, 0\right)\right]^{-1} z, \quad z=\int_{0}^{T_{0}} S\left(T_{0}, \theta\right) g_{u}(\theta) d \theta+\sum_{0 \leq \tau_{k}<T_{0}} S\left(t, \tau_{k}^{+}\right) c_{k} .
$$

Further,

$$
P: L_{T_{0}}^{p}([0, \infty) ; X) \times X^{\delta} \longrightarrow P C_{T_{0}}([0, \infty) ; X)
$$

is a bounded linear operator and

$$
\left\|P\left(g_{u}, c_{k}\right)\right\|_{P C_{T_{0}}([0, \infty) ; X)} \leq \widetilde{B}\left(\|f\|_{L_{T_{0}}^{p}}+\|C\|_{\infty}\|u\|_{L_{T_{0}}^{p}}+\sum_{k=1}^{\delta}\left\|c_{k}\right\|\right),
$$

where $\widetilde{B}=K(K\|Q\|+1)$ and $Q=\left[I-S\left(T_{0}, 0\right)\right]^{-1}$.

Further, for arbitrary $x_{0} \in X$, the PC-mild solution $x\left(\cdot, x_{0}, u\right)$ of the Cauchy problem (3.2) corresponding to the initial value $x_{0} \in X$ and control $u \in U_{a d}$, satisfies the following inequality:

$$
\left\|x\left(t, x_{0}, u\right)-x_{T_{0}}(t, \bar{x}, u)\right\| \leq \widehat{B} e^{-v t}\left(\|f\|_{L_{T_{0}}^{p}}+\|C\|_{\infty}\|u\|_{L_{T_{0}}^{p}}+\sum_{k=1}^{\delta}\left\|c_{k}\right\|\right)
$$

where $x_{T_{0}}(\cdot, \bar{x}, u)$ is the $T_{0}$-periodic PC-mild solution of system (3.1), $\widehat{B}>0$ is not dependent on $x_{0}$, $f, u$, and $c_{k}$. That is, $x\left(\cdot, x_{0}, u\right)$ can be approximated to the $T_{0}$-periodic PC-mild solution $x_{T_{0}}(\cdot, \bar{x}, u)$ according to exponential decreasing speed.

Proof. Consider the operator $Q=\sum_{n=0}^{\infty}\left[S\left(T_{0}, 0\right)\right]^{n}$. By (4) of Lemma 2.1 and the stability of $\{S(\cdot, \cdot)\}$, we have

$$
\left\|\left[S\left(T_{0}, 0\right)\right]^{n}\right\|=\left\|S\left(n T_{0}, 0\right)\right\| \leq K e^{-v n T_{0}} \longrightarrow 0 \quad \text { as } n \longrightarrow \infty .
$$

Thus, $\|Q\| \leq \sum_{n=0}^{\infty}\left\|\left[S\left(T_{0}, 0\right)\right]^{n}\right\| \leq \sum_{n=0}^{\infty} K e^{-v n T_{0}}$. Obviously, the series $\sum_{n=0}^{\infty} K e^{-v n T_{0}}$ is convergent, thus operator $Q \in £_{b}(X)$. It comes from $\left[I-S\left(T_{0}, 0\right)\right] Q=Q\left[I-S\left(T_{0}, 0\right)\right]=I$ that $Q=\left[I-S\left(T_{0}, 0\right)\right]^{-1} \in £_{b}(X)$. It is well known that system (3.1) has a periodic PC-mild solution if and only if $x\left(T_{0}\right)=x(0)$. Since $I-S\left(T_{0}, 0\right)$ is invertible, we can uniquely solve

$$
x(0)=\left[I-S\left(T_{0}, 0\right)\right]^{-1}\left[\int_{0}^{T_{0}} S\left(T_{0}, \theta\right) g_{u}(\theta) d \theta+\sum_{0 \leq \tau_{k}<T_{0}} S\left(t, \tau_{k}^{+}\right) c_{k}\right] .
$$

Let $\bar{x}=\left[I-S\left(T_{0}, 0\right)\right]^{-1} z$, where

$$
z=\int_{0}^{T_{0}} S\left(T_{0}, \theta\right) g_{u}(\theta) d \theta+\sum_{0 \leq \tau_{k}<T_{0}} S\left(t, \tau_{k}^{+}\right) c_{k}
$$


JinRong Wang et al.

Note that

$$
\int_{T_{0}}^{t+T_{0}} S\left(t+T_{0}, s\right) C(s) u(s) d s=\int_{0}^{t} S(t, s) C(s) u(s) d s
$$

it is not difficult to verify that the $P C$-mild solution of the Cauchy problem (3.2) corresponding to initial value $x(0)=\bar{x}$ given by

$$
x(t, u)=S(t, 0)\left[I-S\left(T_{0}, 0\right)\right]^{-1} z+\int_{0}^{t} S(t, \theta) g_{u}(\theta) d \theta+\sum_{0 \leq \tau_{k}<t} S\left(t, \tau_{k}^{+}\right) c_{k}
$$

is just the unique $T_{0}$-periodic of system (3.1).

It is obvious that $P: L_{T_{0}}^{p}([0, \infty) ; X) \times X^{\delta} \rightarrow P C_{T_{0}}([0, \infty) ; X)$ is linear. Next, verify the estimation (3.8). In fact, for $t \in\left[0, T_{0}\right]$,

$$
\left\|x_{T_{0}}(t, \bar{x}, u)\right\| \leq\|S(t, 0)\|\|\bar{x}\|+\int_{0}^{t}\|S(t, \theta)\|\left\|g_{u}(\theta)\right\| d \theta+\sum_{0 \leq \tau_{k}<t}\left\|S\left(t, \tau_{k}^{+}\right)\right\|\left\|c_{k}\right\| .
$$

On the other hand,

$$
\begin{aligned}
\|\bar{x}\| & \leq\left\|\left[I-S\left(T_{0}, 0\right)\right]^{-1}\right\|\left[\int_{0}^{T_{0}}\left\|S\left(T_{0}, \theta\right)\right\|\left\|g_{u}(\theta)\right\| d \theta+\sum_{0 \leq \tau_{k}<T_{0}}\left\|S\left(T_{0}, \tau_{k}^{+}\right)\right\|\left\|c_{k}\right\|\right] \\
& \leq\|Q\|\left[\int_{0}^{T_{0}} K e^{-v\left(T_{0}-\theta\right)}\left\|g_{u}(\theta)\right\| d \theta+K e^{-v\left(T_{0}-\tau_{k}^{+}\right)} \sum_{k=1}^{\delta}\left\|c_{k}\right\|\right] \\
& \leq K\|Q\|\left(\|f\|_{L_{T_{0}}^{p}}+\|C\|_{\infty}\|u\|_{L_{T_{0}}^{p}}+\sum_{k=1}^{\delta}\left\|c_{k}\right\|\right) .
\end{aligned}
$$

Let $\widetilde{B}=K(K\|Q\|+1)$, next the estimation (3.8) is verified.

System (3.1) has a unique $T_{0}$-periodic $P C$-mild solution $x_{T_{0}}(\cdot, \bar{x}, u)$ given by (3.5) and (3.6). The $P C$-mild solution $x\left(\cdot, x_{0}, u\right)$ of the Cauchy problem (3.2) corresponding to initial value $x_{0}$ and control $u \in U_{a d}$ can be given by (3.4). Then,

$$
\begin{aligned}
\left\|x\left(t, x_{0}, u\right)-x_{T_{0}}(t, \bar{x}, u)\right\| & \leq\left\|S(t, 0)\left(\bar{x}-x_{0}\right)\right\| \leq K e^{-v t}\left(\left\|x_{0}\right\|+\|\bar{x}\|\right) \\
& \leq K e^{-v t}\left[\left\|x_{0}\right\|+K\|Q\|\left(\|f\|_{L_{T_{0}}^{1}}+\|C\|_{\infty}\|u\|_{L_{T_{0}}^{P}}+\sum_{k=1}^{\delta}\left\|c_{k}\right\|\right)\right] .
\end{aligned}
$$

Let $\widehat{B}=\max \left\{K, K^{2}\|Q\|\right\}>0$, one can obtain (3.9) immediately. 
Definition 3.3. The $T_{0}$-periodic $P C$-mild solution $x_{T_{0}}(\cdot, \bar{x}, u)$ of the system (3.1) is said to be globally asymptotically stable in the sense that

$$
\lim _{t \rightarrow+\infty}\left\|x\left(t, x_{0}, u\right)-x_{T_{0}}(t, \bar{x}, u)\right\|=0,
$$

where $x\left(\cdot, x_{0}, u\right)$ is any $P C$-mild solutions of the Cauchy problem (3.2) corresponding to initial value $x_{0} \in X$ and control $u \in U_{a d}$.

By Theorem 3.2 and the stability of the impulsive evolution operator $\{S(\cdot, \cdot)\}$ in Section 2 , one can obtain the following results.

Corollary 3.4. Under the assumptions of Theorem 3.2, the system (3.1) has a unique $T_{0}$-periodic PC-mild solution $x_{T_{0}}(\cdot, \bar{x}, u)$ which is globally asymptotically stable.

\section{Existence of periodic optimal harvesting policy}

In this section, we discuss existence of periodic optimal harvesting policy, that is, periodic optimal controls for optimal control problems arising in systems governed by linear impulsive periodic system on Banach space.

By the $T_{0}$-periodic $P C$-mild solution expression of system (3.1) given in Theorem 3.2, one can obtain the result.

Theorem 4.1. Under the assumptions of Theorem 3.2, the $T_{0}$-periodic PC-mild solution of system (3.1) continuously depends on the control on $L^{p}\left(\left[0, T_{0}\right] ; Y\right)$, that is, let $x^{1}\left(x^{2}\right)$ be $T_{0}$-periodic PCmild solution of system (3.1) corresponding to $u_{1}\left(u_{2}\right) \in U_{a d} \subseteq L^{p}\left(\left[0, T_{0}\right] ; Y\right)$. There exists constant $\widetilde{K}>0$ such that

$$
\left\|x^{1}-x^{2}\right\|_{P C\left(\left[0, T_{0}\right] ; X\right)} \leq \tilde{K}\left\|u_{1}-u_{2}\right\|_{L^{p}\left(\left[0, T_{0}\right] ; Y\right)} \text {. }
$$

Proof. Since $x^{1}$ and $x^{2}$ are the $T_{0}$-periodic $P C$-mild solution corresponding to $u_{1}$ and $u_{2} \in U_{a d}$, respectively, then we have

$$
x^{i}(t) \equiv x\left(t, u_{i}\right)=S(t, 0)\left[I-S\left(T_{0}, 0\right)\right]^{-1} z_{i}+\int_{0}^{t} S(t, \theta)\left[f(\theta)+C(\theta) u_{i}(\theta)\right] d \theta, \quad i=1,2,
$$

where

$$
z_{i}=\int_{0}^{T_{0}} S\left(T_{0}, \theta\right)\left[f(\theta)+C(\theta) u_{i}(\theta)\right] d \theta, \quad i=1,2 .
$$

Further,

$$
\begin{aligned}
\left\|x^{1}(t)-x^{2}(t)\right\| & \leq\left(\left\|S(t, 0)\left[I-S\left(T_{0}, 0\right)\right]^{-1}\right\|+1\right) \int_{0}^{T_{0}}\left\|S\left(T_{0}, \theta\right)\right\|\|C\|_{\infty}\left\|u_{1}(\theta)-u_{2}(\theta)\right\| d \theta \\
& \leq \tilde{K}\left\|u_{1}-u_{2}\right\|_{L^{p}\left(\left[0, T_{0}\right] ; Y\right)^{\prime}}
\end{aligned}
$$

where $\tilde{K}=M_{T_{0}}\left(M_{T_{0}}\|Q\|+1\right)\|C\|_{\infty}$. This completes the proof. 
Lemma 4.2. Suppose $C$ is a strong continuous operator. The operator $\Theta: L_{p}\left(\left[0, T_{0}\right] ; Y\right) \rightarrow$ $P C\left(\left[0, T_{0}\right] ; X\right)$, given by

$$
(\Theta u)(\cdot)=\int_{0}^{\cdot} S(\cdot, \theta) C(\theta) u(\theta) d \theta
$$

is strongly continuous.

Proof. Without loss of generality, for $\tau_{k-1} \leq s<\tau_{k}<t \leq \tau_{k+1}$,

$$
(\Theta u)(t)=\int_{0}^{t} T\left(t-\tau_{k}^{+}\right)\left[\prod_{\theta<\tau_{j}<t}\left(I+B_{j}\right) \cdot T\left(\tau_{j}-\tau_{j-1}^{+}\right)\left(I+B_{i}\right) T\left(\tau_{i}-\theta\right) C(s) u(s)\right] d s .
$$

By virtue of strong continuity of $C$, boundedness of $B_{k}, \sup _{t \in\left[0, T_{0}\right]}\|T(t)\|=\mathcal{C}_{T_{0}}<\infty, \Theta$ is strongly continuous.

Let $x(\cdot, u)$ denote the $T_{0}$-periodic PC-mild solution of system (3.1) corresponding to the control $u \in U_{a d}$, we consider the Bolza problem (P).

Find $u^{0} \in U_{a d}$ such that $J\left(u^{0}\right) \leq J(u)$, for all $u \in U_{a d}$, where

$$
J(u)=\int_{0}^{T_{0}} l(t, x(t, u), u(t)) d t+\Psi\left(x\left(T_{0}, u\right)\right)
$$

We introduce the following assumption on $l$ and $\Psi$.

Assumption [H7].

[H7.1] The functional $l:\left[0, T_{0}\right] \times X \times Y \rightarrow R \cup\{\infty\}$ is Borel measurable.

[H7.2] $l(t, \cdot, \cdot)$ is sequentially lower semicontinuous on $X \times Y$ for almost all $t \in\left[0, T_{0}\right]$.

[H7.3] $l(t, x, \cdot)$ is convex on $Y$ for each $x \in X$ and almost all $t \in\left[0, T_{0}\right]$.

[H7.4] There exist constants $d \geq 0, e>0, \varphi$ is nonnegative and $\varphi \in L^{1}\left(\left[0, T_{0}\right] ; \mathbb{R}\right)$ such that

$$
l(t, x, u) \geq \varphi(t)+d\|x\|+e\|u\|_{\gamma}^{p}
$$

[H7.5] The functional $\Psi \rightarrow \mathbb{R}$ is continuous and nonnegative.

Now we can give the following results on existence of periodic optimal controls for Bolza problem $(\mathrm{P})$.

Theorem 4.3. Suppose $C$ is a strong continuous operator and assumption [H7] holds. Under the assumptions of Theorem 3.2, the problem $(P)$ has a unique solution.

Proof. If $\inf \left\{J(u) \mid u \in U_{a d}\right\}=+\infty$, there is nothing to prove. 
We assume that $\inf \left\{J(u) \mid u \in U_{a d}\right\}=\rho<+\infty$. By assumption [H7], we have

$$
J(u) \geq \int_{0}^{T_{0}} \varphi(t) d t+d \int_{0}^{T_{0}}\|x(t)\| d t+e \int_{0}^{T_{0}}\|u(t)\|_{Y}^{p} d t+\Psi\left(x\left(T_{0}, u\right)\right) \geq-\eta>-\infty,
$$

where $\eta>0$ is a constant. Hence $\rho \geq-\eta>-\infty$.

By the definition of infimum there exists a sequence $\left\{u_{n}\right\} \subset U_{a d} \subseteq L^{p}\left(\left[0, T_{0}\right], Y\right)(1<$ $p<\infty)$, such that $\lim _{n \rightarrow \infty} J\left(u_{n}\right)=\rho$.

Since $\left\{u_{n}\right\}$ is bounded in $L^{p}\left(\left[0, T_{0}\right] ; Y\right)(1<p<\infty)$, there exists a subsequence, relabeled as $\left\{u_{n}\right\}$, and $u^{0} \in U$ such that $\lim _{n \rightarrow \infty} u_{n}=u^{0}$ weakly convergence in $L^{p}\left(\left[0, T_{0}\right] ; Y\right)$, and $J\left(u_{n}\right)<\rho+\varepsilon$. Because of $U_{a d}$ is the closed and convex set, thanks to the Mazur lemma, $u^{0} \in U_{a d}$. Suppose $x\left(\cdot, u_{n}\right)$ and $x\left(\cdot, u^{0}\right)$ are the $T_{0}$-periodic PC-mild solution of system (3.1) corresponding to $u_{n}(n=1,2, \ldots)$ and $u^{0}$, respectively, then $x\left(\cdot, u_{n}\right)$ and $x\left(\cdot, u^{0}\right)$ can be given by

$$
\begin{aligned}
& x_{n}(t) \equiv x\left(t, u_{n}\right)=S(t, 0)\left[I-S\left(T_{0}, 0\right)\right]^{-1} z_{n}+\int_{0}^{t} S(t, \theta) g_{u_{n}}(\theta) d \theta+\sum_{0 \leq \tau_{k}<t} S\left(t, \tau_{k}^{+}\right) c_{k}, \\
& x^{0}(t) \equiv x\left(t, u^{0}\right)=S(t, 0)\left[I-S\left(T_{0}, 0\right)\right]^{-1} z_{0}+\int_{0}^{t} S(t, \theta) g_{u^{0}}(\theta) d \theta+\sum_{0 \leq \tau_{k}<t} S\left(t, \tau_{k}^{+}\right) c_{k},
\end{aligned}
$$

where

$$
\begin{aligned}
& z_{n}=\int_{0}^{T_{0}} S\left(T_{0}, \theta\right) g_{u_{n}}(\theta) d \theta+\sum_{0 \leq \tau_{k}<T_{0}} S\left(t, \tau_{k}^{+}\right) c_{k}, \\
& z_{0}=\int_{0}^{T_{0}} S\left(T_{0}, \theta\right) g_{u^{0}}(\theta) d \theta+\sum_{0 \leq \tau_{k}<T_{0}} S\left(t, \tau_{k}^{+}\right) c_{k} .
\end{aligned}
$$

Define

$$
\eta_{n}(t)=\left(\Theta u_{n}\right)(t)-\left(\Theta u^{0}\right)(t)=\int_{0}^{t} S(t, \theta) C(\theta)\left[u_{n}(\theta)-u^{0}(\theta)\right] d \theta
$$

then by Lemma 4.2, we have

$$
\eta_{n} \longrightarrow 0 \text { in } P C\left(\left[0, T_{0}\right] ; X\right) \text { with strongly convergence, }
$$

as $u_{n} \stackrel{w}{\rightarrow} u^{0}$ weakly convergence in $L^{p}\left(\left[0, T_{0}\right] ; Y\right)$.

Next, we show that

$$
x_{n} \longrightarrow x^{0} \text { in } \operatorname{PC}\left(\left[0, T_{0}\right] ; X\right) \text { with strongly convergence as } n \longrightarrow \infty \text {. }
$$


In fact, for $t \in\left[0, \tau_{1}\right]$, we have

$$
\left\|x_{n}(t)-x^{0}(t)\right\| \leq\left(M_{T_{0}}\|Q\|+1\right)\left\|\eta_{n}(t)\right\|_{C\left(\left[0, \tau_{1}\right] ; X\right)} \leq C_{1}\left\|\eta_{n}\right\|_{C\left(\left[0, \tau_{1}\right] ; X\right)} .
$$

By elementary computation, we arrive at

$$
\| x_{n}\left(\tau_{1}^{+}-x^{0}\left(\tau_{1}^{+}\right)\left\|\leq\left(\left\|B_{1}\right\|+1\right)\right\| x_{n}\left(\tau_{1}\right)-x^{0}\left(\tau_{1}\right)\left\|\leq C_{1}^{\prime}\right\| \eta_{n} \|_{C\left(\left[0, \tau_{1}\right] ; X\right)}\right.
$$

Consider the time interval $\left(\tau_{1}, \tau_{2}\right]$, similarly we obtain

$$
\left\|x_{n}(t)-x^{0}(t)\right\| \leq C_{2}\left\|\eta_{n}\right\|_{C\left(\left(\tau_{1}, \tau_{2}\right], X\right)^{\prime}} \quad\left\|x_{n}\left(\tau_{2}^{+}\right)-x^{0}\left(\tau_{2}^{+}\right)\right\| \leq C_{2}^{\prime}\left\|\eta_{n}\right\|_{C\left(\left(\tau_{1}, \tau_{2}\right] ; X\right)} .
$$

In general, given any $\tau_{k} \in \tilde{D}, k=1,2, \ldots, n$, and the $x_{n}\left(\tau_{k}\right), x^{0}\left(\tau_{k}\right)$, prior to the jump at time $\tau_{k}$, we immediately follow the jump as

$$
x_{n}\left(\tau_{k}^{+}\right)=x_{n}\left(\tau_{k}\right)+B_{k} x_{n}\left(\tau_{k}\right)+c_{k}, \quad x^{0}\left(\tau_{k}^{+}\right)=x^{0}\left(\tau_{k}\right)+B_{k} x^{0}\left(\tau_{k}\right)+c_{k},
$$

the associated interval $\left(\tau_{k}, \tau_{k+1}\right]$, we also similarly obtain

$$
\left\|x_{n}(t)-x^{0}(t)\right\| \leq C_{k+1}\left\|\eta_{n}\right\|_{C\left(\left[\tau_{k}, \tau_{k+1}\right] ; X\right)^{\prime}} \quad\left\|x_{n}\left(\tau_{k+1}^{+}\right)-x^{0}\left(\tau_{k+1}^{+}\right)\right\| \leq C_{k+1}^{\prime}\left\|\eta_{n}\right\|_{C\left(\left[\tau_{k}, \tau_{k+1}\right] ; X\right)}
$$

Step by step, we repeat the procedures till the time interval is exhausted. Let $\widehat{C}=$ $\max \left\{C_{1}, C_{1}^{\prime}, C_{2}, C_{2}^{\prime}, \ldots, C_{k+1}\right\}$, thus we obtain

$$
\left\|x_{n}-x^{0}\right\|_{P C\left(\left[0, T_{0}\right] ; X\right)} \leq \hat{\mathcal{C}}\left\|\eta_{n}\right\|_{P C\left(\left[0, T_{0}\right] ; X\right)}
$$

that is,

$$
x_{n} \longrightarrow x^{0} \text { in } \operatorname{PC}\left(\left[0, T_{0}\right] ; X\right),
$$

with strongly convergence as $n \rightarrow \infty$.

Since $P C\left(\left[0, T_{0}\right] ; X\right) \hookrightarrow L^{1}\left(\left[0, T_{0}\right] ; X\right)$, using the assumption [H7] and Balder's theorem, we can obtain

$$
\rho=\lim _{n \rightarrow \infty} \int_{0}^{T_{0}} l\left(t, x_{n}(t), u_{n}(t)\right) d t+\Psi\left(x_{n}\left(T_{0}\right)\right) \geq \int_{0}^{T_{0}} l\left(t, x^{0}(t), u^{0}(t)\right) d t+\Psi\left(x^{0}\left(T_{0}\right)\right) \geq \rho .
$$

This shows that $J$ attains its minimum at $u^{0} \in U_{a d}$. This completes the proof. 


\section{Example}

Last, an academic example is given to illustrate our theory.

Let $X=U=Y=L^{2}(0,1)$ and consider the following population evolution equation with impulses:

$$
\begin{gathered}
\frac{\partial}{\partial t} x(t, y)+a \frac{\partial}{\partial y} x(t, y)=k x(t, y)+b \sin (t, y)+u(t, y), \\
u \in U_{a d} \subseteq U, y \in(0,1), t \in(0,2 k \pi] \backslash\left\{\frac{1}{2} \pi, \pi, \frac{3}{2} \pi, \ldots\right\}, \quad k \in \mathbb{Z}_{0}^{+}, \\
x(0, y)=x(2 \pi, y)=0, \quad y \in(0,1), \\
x(t, 0)=x(t, 1)=0, \quad t>0, \\
\Delta x\left(\tau_{k}, y\right)=\left\{\begin{array}{l}
0.05 I x\left(\tau_{k}, y\right), \quad k=1, \\
-0.05 I x\left(\tau_{k}, y\right), \quad k=2, y \in(0,1), t>0, \\
0.05 I x(t, y), \quad k=3,
\end{array}\right.
\end{gathered}
$$

where $t$ denotes time, $y$ denotes age, $x(t, y)$ is called age density function, $a$ and $b$ are positive constants, $k$ is a bounded measurable function, that is, $k \in L^{\infty}(0,1)$. $k$ denotes the age-specific death rate, $b \sin (t, y)$ denotes the age density of migrants, and $u(t, y)$ denotes the control. The admissible control set $U_{a d}=\left\{u \in Y \mid\|u\|_{L^{2}\left(\left[0, T_{0}\right] ; Y\right)} \leq 1\right\}$.

A linear operator $A$ defined on $X$ by

$$
A x=-a \frac{d x}{d y}+k x, \quad \forall x \in D(A)
$$

where the domain of $A$ is given by

$$
D(A)=\left\{x \in X: x \text { is a absolutely continuous, } \frac{d x}{d y} \in X, \quad x(0)=x(2 \pi)=0\right\} .
$$

By the fact that the operator $-(d \cdot / d y)$ is an infinitesimal generator of a $C_{0}$-semigroup (see [39, Example 2.21]) and [38, Theorem 4.2.1], then $A$ is an infinitesimal generator of a $C_{0^{-}}$ semigroup since the operator $k I$ is bounded.

Now let us consider the following operators family:

$$
(T(t) x)(y)=\left\{\begin{array}{l}
\exp \left(\frac{1}{a} \int_{y-a t}^{y} k(s) d s\right) \cdot x(y-a t), \quad \text { if } 0 \leq t \leq \frac{y}{a} \\
0, \quad \text { otherwise. }
\end{array}\right.
$$

It is not difficult to verify that $\{T(t), t \geq 0\}$ defines a $C_{0}$-semigroup and $A$ is just the infinitesimal generator of the $C_{0}$-semigroup $\{T(t), t \geq 0\}$. Since $k \in L^{\infty}(0,1)$, then there exits 
a constant $m>0$ such that $|k(s)| \leq m$ a.e. $s \in[0,1]$. For an arbitrary function $x \in L^{2}(0,1)$, by using the expression (5.4) of the semigroup $\{T(t), t \geq 0\}$, the following inequality holds:

$$
\begin{aligned}
\int_{0}^{\infty}\|T(t) x\|^{2} d t & \leq \int_{0}^{y / a} \exp \left(2\left\|\frac{1}{a} \int_{y-a t}^{y} m d s\right\|\right) d s \cdot\|x\|_{L^{2}(0,1)}^{2} \\
& \leq \int_{0}^{1 / a} \exp (2 m t) d s \cdot\|x\|_{L^{2}(0,1)}^{2} \\
& \leq \frac{\exp \left(2 m a^{-1}\right)-1}{2 m}\|x\|_{L^{2}(0,1)}^{2} .
\end{aligned}
$$

Hence, Lemma 2.3 leads to the exponential stability of $\{T(t), t \geq 0\}$. That is, there exist $K_{0}>0$ and $v_{0}>0$ such that $\|T(t)\| \leq K_{0} e^{-v_{0} t}, t>0$.

Let

$$
\widetilde{J}(u)=\int_{0}^{2 \pi} \int_{0}^{1}\left(|x(t, \xi)|^{2}+|u(t, \xi)|^{2}\right) d \xi d t+\int_{0}^{1}|x(2 \pi, \xi)|^{2} d \xi
$$

Define $x(\cdot)(y)=x(\cdot, y), \sin (\cdot)(y)=\sin (\cdot, y), B(\cdot) u(\cdot)(y)=u(\cdot, y), B_{1}=B_{3}=0.05 I$, $B_{2}=-0.05 I$. Thus system (5.1) can be rewritten as

$$
\begin{gathered}
\dot{x}(t)=A x(t)+b \sin t+u(t), \quad u \in U_{a d}, t \in(0,2 k \pi] \backslash\left\{\frac{1}{2} \pi, \pi, \frac{3}{2} \pi, \ldots\right\}, \quad k \in \mathbb{Z}_{0}^{+}, \\
\Delta x\left(\frac{i}{2} \pi\right)=B_{i} x\left(\frac{i}{2} \pi\right), \quad i=1,2,3, \ldots,
\end{gathered}
$$

with the cost function

$$
J(u)=\int_{0}^{2 \pi}\left(\|x(t)\|^{2}+\|u(t)\|^{2}\right) d t+\|x(2 \pi)\|^{2} .
$$

By Lemma 2.4, for $v_{0}>\lambda>3 \ln K_{0}+2 \ln 1.05+\ln 0.95,\{S(t, \theta), t \geq \theta \geq 0\}$ is exponentially stable. Now, all the assumptions are met in Theorems 3.2 and 4.3 , our results can be used to system (5.1). Thus, system (5.1) has a unique $2 \pi$-periodic $P C$-mild solution $x_{2 \pi}(\cdot, y, u) \in P C_{2 \pi}\left([0+\infty) ; L^{2}(0,1)\right)$ which is globally asymptotically stable and there exists a periodic control $u^{0} \in U_{a d}$ such that $J\left(u^{0}\right) \leq J(u)$ for all $u \in U_{a d}$.

The results show that the optimal population level is truly the periodic solution of the considered system, and hence, it is globally asymptotically stable. Meanwhile, it implies that we can achieve sustainability at a high level of productivity and good economic profit by virtue of scientific, effective, and continuous management of the resources.

\section{Acknowledgments}

This work is supported by Natural Science Foundation of Guizhou Province Education Department (no. 2007008). This work is also supported by the undergraduate carve out project of Department of Guiyang Science and Technology (2008, no. 15-2). 


\section{References}

[1] C. W. Clark, Mathematical Bioeconomics: The Optimal Management of Renewable Resources, Pure and Applied Mathematics, John Wiley \& Sons, New York, NY, USA, 1976.

[2] X. Song and L. Chen, "Optimal harvesting and stability for a two-species competitive system with stage structure," Mathematical Biosciences, vol. 170, no. 2, pp. 173-186, 2001.

[3] R. Marzollo, Ed., Periodic Optimization, Springer, New York, NY, USA, 1972.

[4] M. Fan and K. Wang, "Optimal harvesting policy for single population with periodic coefficients," Mathematical Biosciences, vol. 152, no. 2, pp. 165-177, 1998.

[5] D. D. Bainov and P. S. Simeonov, Impulsive Differential Equations: Periodic Solutions and Applications, vol. 66 of Pitman Monographs and Surveys in Pure and Applied Mathematics, Longman Scientific \& Technical, Harlow, UK, 1993.

[6] Y. N. Xiao, D. Z. Cheng, and H. S. Qin, "Optimal impulsive control in periodic ecosystem," Systems E Control Letters, vol. 55, no. 7, pp. 558-565, 2006.

[7] V. Lakshmikantham, D. D. Bainov, and P. S. Simeonov, Theory of Impulsive Differential Equations, vol. 6 of Series in Modern Applied Mathematics, World Scientific, Teaneck, NJ, USA, 1989.

[8] J. J. Nieto, “An abstract monotone iterative technique," Nonlinear Analysis: Theory, Methods $\mathcal{E}$ Applications, vol. 28, no. 12, pp. 1923-1933, 1997.

[9] J. Yan, A. Zhao, and J. J. Nieto, "Existence and global attractivity of positive periodic solution of periodic single-species impulsive Lotka-Volterra systems," Mathematical and Computer Modelling, vol. 40, no. 5-6, pp. 509-518, 2004.

[10] J.-J. Jiao, L.-S. Chen, J. J. Nieto, and T. Angela, "Permanence and global attractivity of stage-structured predator-prey model with continuous harvesting on predator and impulsive stocking on prey," Applied Mathematics and Mechanics, vol. 29, no. 5, pp. 653-663, 2008.

[11] G. Zeng, F. Wang, and J. J. Nieto, "Complexity of a delayed predator-prey model with impulsive harvest and Holling type II functional response," Advances in Complex Systems, vol. 11, no. 1, pp. 7797, 2008.

[12] W. Wang, J. Shen, and J. J. Nieto, "Permanence and periodic solution of predator-prey system with Holling type functional response and impulses," Discrete Dynamics in Nature and Society, vol. 2007, Article ID 81756, 15 pages, 2007.

[13] H. Zhang, L. Chen, and J. J. Nieto, "A delayed epidemic model with stage-structure and pulses for pest management strategy," Nonlinear Analysis: Real World Applications, vol. 9, no. 4, pp. 1714-1726, 2008.

[14] B. Ahmad and J. J. Nieto, "Existence and approximation of solutions for a class of nonlinear impulsive functional differential equations with anti-periodic boundary conditions," Nonlinear Analysis: Theory, Methods E Applications, vol. 69, no. 10, pp. 3291-3298, 2008.

[15] D. Jiang, Y. Yang, J. Chu, and D. O'Regan, "The monotone method for Neumann functional differential equations with upper and lower solutions in the reverse order," Nonlinear Analysis: Theory, Methods E Applications, vol. 67, no. 10, pp. 2815-2828, 2007.

[16] Y. Li, "Global exponential stability of BAM neural networks with delays and impulses," Chaos, Solitons E Fractals, vol. 24, no. 1, pp. 279-285, 2005.

[17] M. De la Sen, "Stability of impulsive time-varying systems and compactness of the operators mapping the input space into the state and output spaces," Journal of Mathematical Analysis and Applications, vol. 321, no. 2, pp. 621-650, 2006.

[18] L. Yu, J. Zhang, Y. Liao, and J. Ding, "Parameter estimation error bounds for Hammerstein nonlinear finite impulsive response models," Applied Mathematics and Computation, vol. 202, no. 2, pp. 472-480, 2008.

[19] T. Jankowski, "Positive solutions to second order four-point boundary value problems for impulsive differential equations," Applied Mathematics and Computation, vol. 202, no. 2, pp. 550-561, 2008.

[20] E. M. Hernández, M. Pierri, and G. Goncalves, "Existence results for an impulsive abstract partial differential equation with state-dependent delay," Computers \& Mathematics with Applications, vol. 52, no. 3-4, pp. 411-420, 2006.

[21] E. M. Hernández, M. Rabello, and H. R. Henríquez, “Existence of solutions for impulsive partial neutral functional differential equations," Journal of Mathematical Analysis and Applications, vol. 331, no. 2, pp. 1135-1158, 2007.

[22] E. M. Hernández, R. Sakthivel, and S. T. Aki, “Existence results for impulsive evolution differential equations with state-dependent delay," Electronic Journal of Differential Equations, vol. 2008, no. 28, pp. $1-11,2008$. 
[23] N. U. Ahmed, "Some remarks on the dynamics of impulsive systems in Banach spaces," Dynamics of Continuous, Discrete and Impulsive Systems. Series A, vol. 8, no. 2, pp. 261-274, 2001.

[24] N. U. Ahmed, K. L. Teo, and S. H. Hou, "Nonlinear impulsive systems on infinite dimensional spaces," Nonlinear Analysis: Theory, Methods E Applications, vol. 54, no. 5, pp. 907-925, 2003.

[25] X. Xiang and N. U. Ahmed, "Existence of periodic solutions of semilinear evolution equations with time lags," Nonlinear Analysis: Theory, Methods \& Applications, vol. 18, no. 11, pp. 1063-1070, 1992.

[26] X. Xiang, "Optimal control for a class of strongly nonlinear evolution equations with constraints," Nonlinear Analysis: Theory, Methods \& Applications, vol. 47, no. 1, pp. 57-66, 2001.

[27] P. Sattayatham, S. Tangmanee, and W. Wei, "On periodic solutions of nonlinear evolution equations in Banach spaces," Journal of Mathematical Analysis and Applications, vol. 276, no. 1, pp. 98-108, 2002.

[28] X. Xiang, W. Wei, and Y. Jiang, "Strongly nonlinear impulsive system and necessary conditions of optimality," Dynamics of Continuous, Discrete E Impulsive Systems. Series A, vol. 12, no. 6, pp. 811-824, 2005.

[29] W. Wei and X. Xiang, "Necessary conditions of optimal control for a class of strongly nonlinear impulsive equations in Banach spaces," Nonlinear Analysis: Theory, Methods \& Applications, vol. 63, no. 5-7, pp. 53-63, 2005.

[30] W. Wei, X. Xiang, and Y. Peng, "Nonlinear impulsive integro-differential equations of mixed type and optimal controls," Optimization, vol. 55, no. 1-2, pp. 141-156, 2006.

[31] X. Xiang and W. Wei, "Mild solution for a class of nonlinear impulsive evolution inclusions on Banach space," Southeast Asian Bulletin of Mathematics, vol. 30, no. 2, pp. 367-376, 2006.

[32] X. Yu, X. Xiang, and W. Wei, "Solution bundle for a class of impulsive differential inclusions on Banach spaces," Journal of Mathematical Analysis and Applications, vol. 327, no. 1, pp. 220-232, 2007.

[33] Y. Peng, X. Xiang, and W. Wei, "Nonlinear impulsive integro-differential equations of mixed type with time-varying generating operators and optimal controls," Dynamic Systems and Applications, vol. 16, no. 3, pp. 481-496, 2007.

[34] Y. Peng and X. Xiang, "Second order nonlinear impulsive time-variant systems with unbounded perturbation and optimal controls," Journal of Industrial and Management Optimization, vol. 4, no. 1, pp. 17-32, 2008.

[35] J. Wang, "Linear impulsive periodic system on Banach spaces," in Proceedings of the 4th International Conference on Impulsive and Hybrid Dynamical Systems, vol. 5, pp. 20-25, Nanning, China, July 2007.

[36] J. Wang, X. Xiang, and W. Wei, "Linear impulsive periodic system with time-varying generating operators on Banach space," Advances in Difference Equations, vol. 2007, Article ID 26196, 16 pages, 2007.

[37] J. Wang, X. Xiang, W. Wei, and Q. Chen, "Existence and global asymptotical stability of periodic solution for the $T$-periodic logistic system with time-varying generating operators and $T_{0}$-periodic impulsive perturbations on Banach spaces," Discrete Dynamics in Nature and Society, vol. 2008, Article ID 524945, 16 pages, 2008.

[38] N. U. Ahmed, Semigroup Theory with Applications to Systems and Control, vol. 246 of Pitman Research Notes in Mathematics Series, Longman Scientific \& Technical, Harlow, UK, 1991.

[39] Z.-H. Luo, B.-Z. Guo, and O. Morgül, Stability and Stabilization of Infinite Dimensional Systems with Applications, Communications and Control Engineering Series, Springer, London, UK, 1999. 